\title{
Open heavy flavor measurements at STAR
}

\author{
David Tlustý ${ }^{1, a}$ \\ ${ }^{1}$ Nuclear Physics Institute, ASCR
}

\begin{abstract}
In relativistic heavy ion collisions at RHIC, heavy quarks are expected to be created from initial hard scatterings. Since heavy quarks have large masses, long life time, and negligible annihilation due to their small population, the number of heavy quarks is conserved during whole medium evolution. The interaction between heavy quarks and the medium is sensitive to the early medium dynamics, therefore heavy quarks are suggested as an ideal probe to quantify the properties of the strongly interacting QCD matter.

In this article, we report on recent STAR results of open heavy flavor production at $\sqrt{s}=$ 200 and $500 \mathrm{GeV}$ in $\mathrm{p}+\mathrm{p}, \sqrt{s_{N N}}=200 \mathrm{GeV}$ in $\mathrm{Au}+\mathrm{Au}$ and $\sqrt{s_{N N}}=193 \mathrm{GeV}$ in $\mathrm{U}+\mathrm{U}$ collisions.
\end{abstract}

\section{Introduction}

In relativistic heavy ion collisions at RHIC, heavy quarks are expected to be created from initial hard scatterings. Since heavy quarks have large masses, long life time, and negligible annihilation due to their small population, the number of heavy quarks is conserved during whole medium evolution. The interaction between heavy quarks and the medium is sensitive to the medium properties, such as the transport coefficients, during its early stages when the QGP phase is expected to exist in heavy-ion collisions [1].

Energetic heavy quarks were predicted to lose less energy than light quarks via gluon radiation when they traverse the QGP owing to the "Dead cone effect" [2]. In contrast, measurements [3-5] show significant suppression at high transverse momentum, $p_{T}$, in central heavy ion collisions, similar to that of light hadrons. This has led to the reconsideration of the effect of heavy-quark collisional energy loss $[6,7]$.

Heavy quarks are expected to hadronize in elementary collisions mainly through hard fragmentation. In high-energy heavy-ion collisions, the large charm-pair abundance could increase the coalescence probability, in particular for $p_{T}<2 \mathrm{GeV} / c$. The coalescence of charm with a light quark from the medium with a large radial flow may introduce a $p_{T}$-dependent enhancement to the observed charmed hadron spectrum compared to that from fragmentation [23, 24]. Furthermore, this may lead to a baryon-to-meson enhancement for charmed hadrons similar to that observed for light-flavor hadrons $[25,27]$.

The state-of-art measurements of inclusive heavy quark production are carried out through two main approaches:

\footnotetext{
ae-mail: tlusty@gmail.com
} 


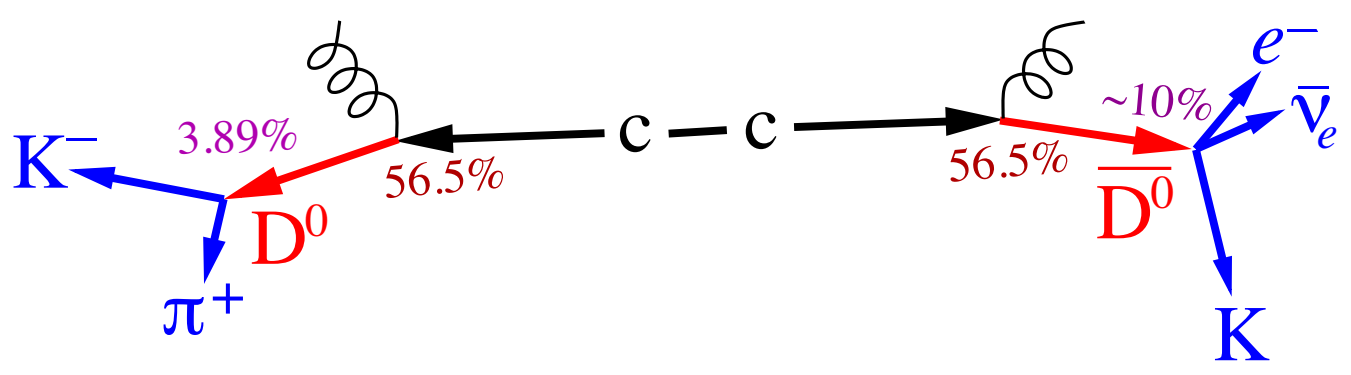

Figure 1. Charm quark fragmentation to $D^{0}$ and two main $D^{0}$ decay channels.

1. single electrons from open heavy hadron semi-leptonic decays

2. hadrons from hadronic decays

Both are drawn schematically in Fig. 1 which depicts a created pair of charm quarks hadronizing into $D^{0}$ and $\overline{D^{0}}$ with the probability of $56.5 \%$. The figure further shows the $D^{0}$ undergoing a hadronic decay into negative kaon and positive pion with branching ratio of $3.87 \%$ and $\overline{D^{0}}$ undergoing a semi-leptonic decay electron, corresponding neutrino and a kaon. The branching ratios as well as the hadronization probability are taken from Ref. [12]. The first approach provides an abundant statistics owing to the variety of decay channels in which those electrons called non-photonic electrons (NPE) are created. The second approach provides direct access to open charm meson kinematics owing to the possibility of the $D^{0}$ invariant mass reconstruction and allows to distinguish an open charm hadron from others.

\section{Analysis Method and Datasets}

Invariant yield of charm quark production $d Y$ is calculated as

$$
d Y \equiv \frac{\mathrm{d}^{2} N_{c \bar{c}}}{2 \pi p_{T} \mathrm{~d} p_{T} \mathrm{~d} y}=\frac{1}{N_{\text {trig }}} \frac{Y\left(p_{T}, y\right)}{2 \pi p_{T} \Delta p_{T} \Delta y} \frac{f_{\text {trg }}}{\Gamma f_{\text {frag }} \epsilon_{\text {rec }}}
$$

where $N_{\text {trig }}$ is the total number of triggered events used for the analysis. $Y\left(p_{T}, y\right)$ is the raw charm hadron signal in each $p_{T}$ bin within a given rapidity window $\Delta y . \Gamma$ is the hadronic decay branching ratio for the channel of interest. $\epsilon_{\text {rec }}$ is the reconstruction efficiency including geometric acceptance, track selection efficiency, particle identification (PID) efficiency, and analysis cut efficiency. $f_{\text {frag }}$ represents the the ratio of charm quarks hadronized to open charm mesons. And $f_{\text {trg }}$ is the correction factor to account for the trigger bias. $f_{\text {trg }}$ is found to be unity in $\mathrm{Au}+\mathrm{Au}, 0.65 \mathrm{in} \mathrm{p}+\mathrm{p}$ collisions at $\sqrt{s}=200 \mathrm{GeV}$ and 0.58 in $\mathrm{p}+\mathrm{p}$ collisions at $\sqrt{s}=500 \mathrm{GeV}$.

$Y\left(p_{T}, y\right)$ is obtained from fitting the invariant mass spectrum (Fig. 2) of open charm mesons through hadronic decays: $D^{0}\left(\overline{D^{0}}\right) \rightarrow K^{\mp} \pi^{ \pm}(\Gamma=3.89 \%)$ and $D^{* \pm} \rightarrow D^{0}\left(\overline{D^{0}}\right) \pi^{ \pm}(\Gamma=67.7 \%) \rightarrow$ $K^{-} \pi^{+} \pi^{ \pm}$(total $\Gamma=2.63 \%$ )

The daughter particles were identified by Time Projection Chamber (TPC) and Time-Of-Flight (TOF) subsystems of the STAR experiment [15] at mid-rapidity $|y|<1$ at $\sqrt{s_{N N}}=200$ and $500 \mathrm{GeV}$.

In years 2009-2012, STAR did not have the capability to reconstruct the secondary vertex of $D^{0}$ decay; one had to calculate the invariant mass of all $K \pi$ pairs coming from the vicinity of the primary 

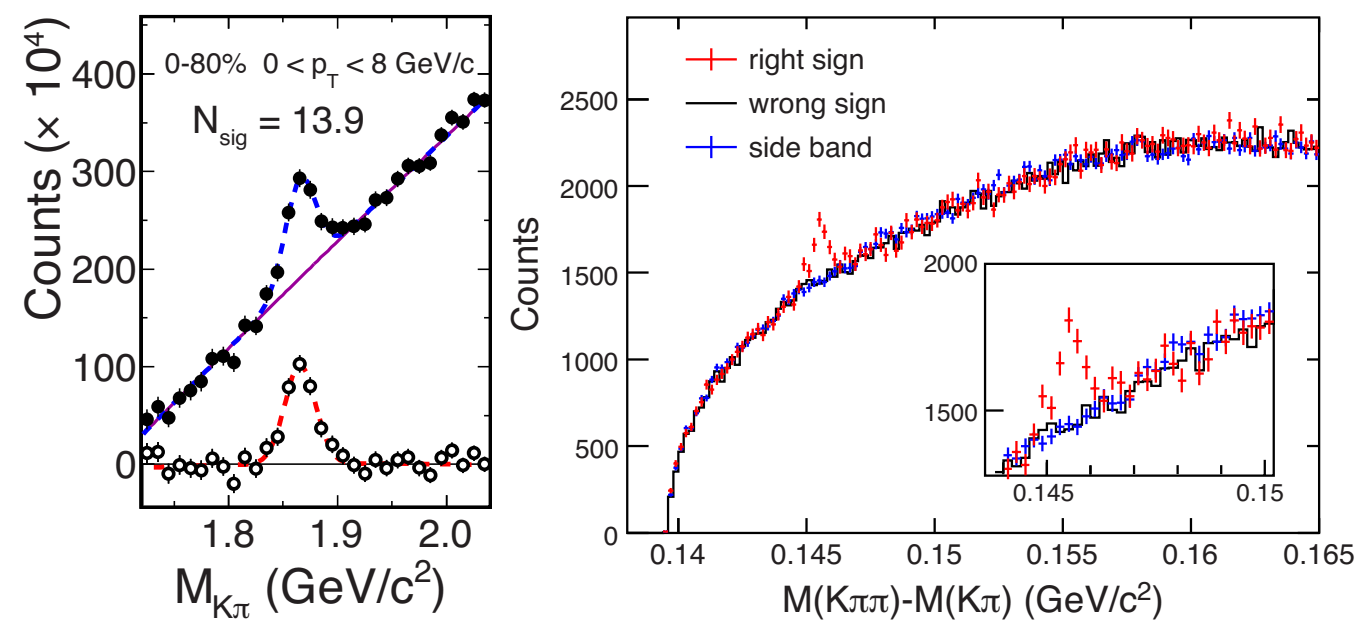

Figure 2. Left panel: $D^{0}$ signal in $\mathrm{Au}+\mathrm{Au} 200 \mathrm{GeV}$ collisions after mixed-event background subtraction. Figure taken from Ref. [13]. Right panel: $D^{*}$ signal in $\mathrm{p}+\mathrm{p} 200 \mathrm{GeV}$ collisions with combinatorial background reproduced by wrong-sign and side-band methods [14].

vertex. This results in a large combinatorial background which was reconstructed via the mixedevent method ( $\mathrm{Au}+\mathrm{Au}$ dataset), same-charge-sign, and kaon momentum-rotation ( $\mathrm{p}+\mathrm{p}$ dataset) and subtracted from invariant mass spectra of all particle pairs [16]. The $D^{* \pm}$ meson undergoes a cascading decay

$$
D^{* \pm} \underset{p^{*}=39 \mathrm{MeV} / \mathrm{c}}{\stackrel{B . R .=67.7 \%}{\longrightarrow}} D^{0} \pi_{S}^{ \pm} \stackrel{B \cdot R \cdot=3.89 \%}{\longrightarrow} K^{\mp} \pi^{ \pm} \pi_{S}^{ \pm}
$$

with very low decay energy giving both daughter particles momentum in CMS of $39 \mathrm{MeV} / c$. Hence the difference in invariant mass

$$
\Delta M \equiv\left(M_{K^{\mp} \pi^{ \pm} \pi^{ \pm}}-M_{K^{\mp} \pi^{ \pm}} ; 1.84<M_{K^{\mp} \pi^{ \pm}}<1.89 \mathrm{GeV} / c^{2}\right)
$$

has very low combinatorial background around $145.4 \mathrm{MeV} / c^{2}$, which is the difference in mass of the $D^{* \pm}$ and the $D^{0}$ meson, and whose resolution is determined by mostly the soft pion $\pi_{S}$ momentum resolution. Both factors imply the ability to have a significant peak in $\Delta M$ spectrum around 145.4 $\mathrm{MeV} / \mathrm{c}^{2}$.

The combinatorial background was reconstructed by side-band (picking $K \pi$ pair outside the $D^{0}$ mass region) and wrong-sign (picking soft pion with opposite charge) methods. The dominant source of systematic uncertainties for both $D^{0}$ and $D^{*}$ analyses was the difference between yields obtained from subtractions of combinatorial background from all particle combinations.

\section{Results}

\subsection{Meson Production in $\mathrm{p}+\mathrm{p}$ Collisions}

Yields $Y\left(p_{T}, y\right)$ were calculated in $p_{T}$ bins and the charm cross section at mid-rapidity $\mathrm{d} \sigma^{c \bar{c}} / \mathrm{d} y$ was obtained from the power-law function

$$
f_{\text {Hagedorn }}\left(p_{T}, y\right)=4 \frac{\mathrm{d} \sigma^{c \bar{c}}}{\mathrm{~d} y} \frac{(n-1)(n-2)}{\left\langle p_{T}\right\rangle^{2}(n-3)^{2}}\left(1+\frac{2 p_{T}}{\left\langle p_{T}\right\rangle(n-3)}\right)^{-n}
$$



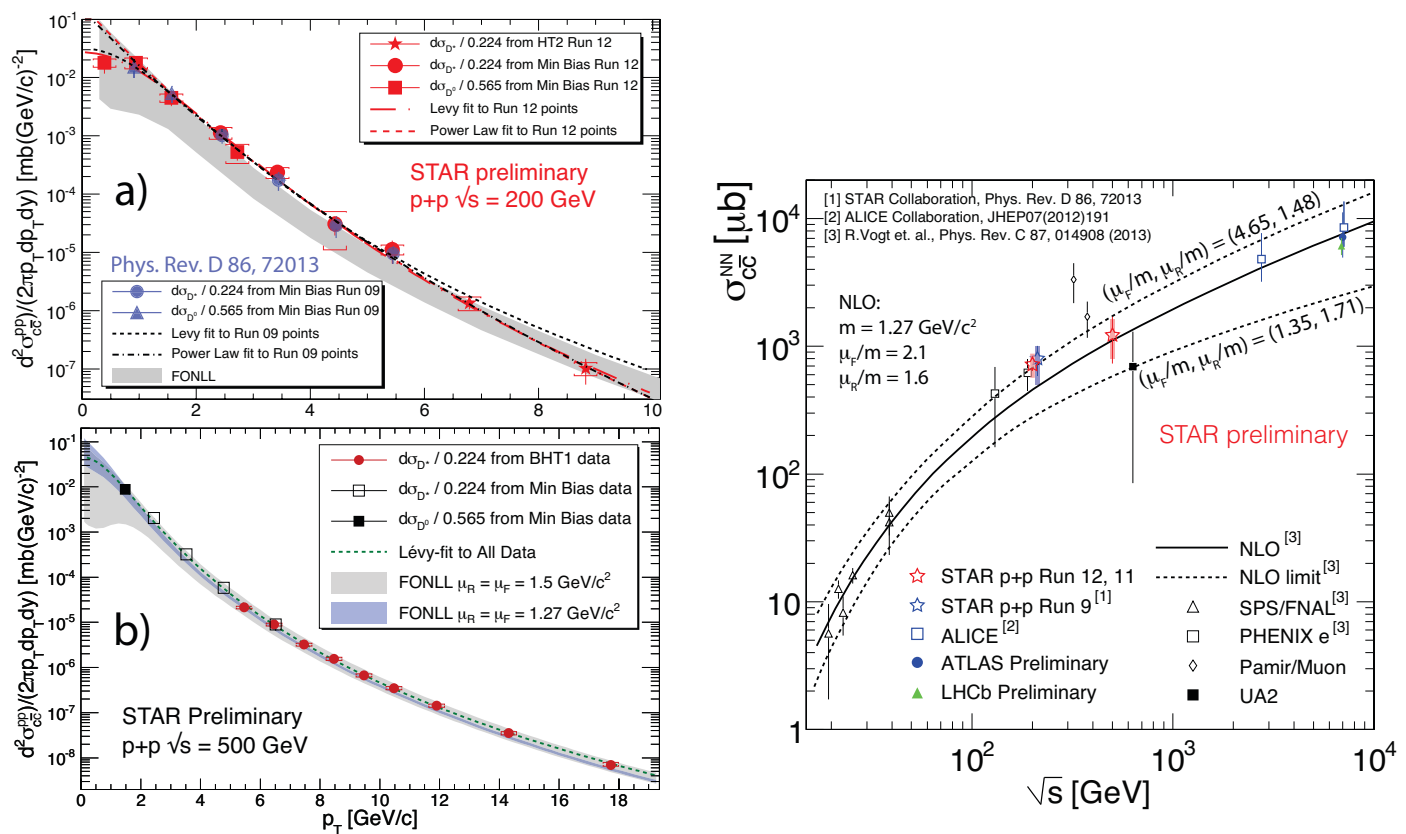

Figure 3. Charm quark production cross section as inferred from $D^{0}$ and $D^{*}$ production in $\mathrm{p}+\mathrm{p}$ collisions Figure 4. Total charm cross section as a function of $\sqrt{s}$. at $\sqrt{s}=200 \mathrm{GeV}$ (panel a) and at $\sqrt{s}=500 \mathrm{GeV}$ (panel b) compared with FONLL prediction (shaded bands).

or

$$
f_{\text {Levy }}\left(p_{T}, y\right)=\frac{\mathrm{d} \sigma_{c \bar{c}}}{\mathrm{~d} y} \frac{(n-1)(n-2)}{2 \pi n C\left[n C+m_{0}(n-2)\right]}\left(1+\frac{\sqrt{p_{T}^{2}+m_{0}^{2}}-m_{0}}{n C}\right)^{-n}
$$

fit [14] to $\mathrm{d}^{2} \sigma^{c \bar{c}} /\left(2 \pi p_{T} \mathrm{~d} p_{T} \mathrm{~d} y\right)=d Y \cdot \sigma_{\mathrm{NSD}}$, where $d Y$ was obtained from (1). $\sigma^{\mathrm{NSD}}$ is the total nonsingle diffractive (NSD) cross section, which is measured at STAR to be $30.0 \pm 2.4 \mathrm{mb}$ at $\sqrt{s}=200$ $\mathrm{GeV}$ [17]. In the case of $\sqrt{s}=500 \mathrm{GeV}$, there is no STAR measurement yet; $\sigma^{\text {NSD }}$ is extrapolated from $200 \mathrm{GeV}$ measurement with the help of PYTHIA simulation to be $34 \mathrm{mb}$.

STAR has published results on $\frac{\mathrm{d}^{2} \sigma^{c \bar{c}}}{2 \pi p_{T} \mathrm{~d} p_{T} \mathrm{~d} y}$ for $0.6<p_{T}<6 \mathrm{GeV} / c$ in $\mathrm{p}+\mathrm{p}$ collisions at $\sqrt{s}=200 \mathrm{GeV}$ [14]. The measurement was done on minimum-bias-triggered (MB) events with data taken in 2009. Recently, a new measurement has been carried out using data taken in $\mathrm{p}+\mathrm{p}$ collisions at $\sqrt{s}=200 \mathrm{GeV}$ in 2012, with both MB events and events triggered on energy deposition in a Barrel ElectroMagnetic Calorimeter (BEMC) tower above certain thresholds, i.e., High- $E_{T}$ triggers (HT). The HT results, after correcting for the trigger efficiency, are consistent with the MB results in the overlapping $p_{T}$ region. As can be seen in panel a) of Fig. 3, the new analysis extends the $p_{T}$ range down to $0 \mathrm{GeV} / c$, thanks to the larger MB data sample size, and up to $10 \mathrm{GeV} / c$, owing to the inclusion of the HT data sample. These new results are consistent with the published ones, and in agreement with fixed order, next-to-leading logarithm (FONLL) pQCD calculations [18]. A similar analysis has also been done using data taken in $\mathrm{p}+\mathrm{p}$ collisions at $\sqrt{s}=500 \mathrm{GeV}$, with both $\mathrm{MB}$ and HT events. The charm 
production cross section is measured as a function of $p_{T}$ for $1<p_{T}<20 \mathrm{GeV} / c$. The results are consistent with the latest FONLL calculations [19], as shown in panel b) of Fig. 3.

In order to compare STAR results with other experiments, we extrapolated $\left.\frac{\mathrm{d} \sigma_{c \bar{c}}}{\mathrm{~d} y}\right|_{y=0}$ to $\sigma_{c \bar{c}}$ using PYTHIA simulations with various parameter tunings giving extrapolation factors $4.7 \pm 0.7$ for 200 $\mathrm{GeV}$ and $5.6 \pm 0.1$ for $500 \mathrm{GeV}$ collisions. The results are shown in Fig. 4.

\section{2 $D^{0}$ Production in Heavy Ion Collisions}

Yields $Y\left(p_{T}, y, N_{\text {bin }}\right)$ were calculated in $p_{T}$ and centrality bins. The charm pair per nucleon-nucleoncollision production cross section $\mathrm{d} \sigma_{c \bar{c}}^{\mathrm{NN}} / \mathrm{d} y$ was obtained from the integral of

$$
\frac{\mathrm{d}^{2} \sigma_{c \bar{c}}^{\mathrm{NN}}}{2 \pi p_{T} \mathrm{~d} p_{T} \mathrm{~d} y}=d Y \cdot \frac{\sigma^{\text {inel }}}{N_{\text {bin }}}
$$

over $p_{T}$ and is measured to be $\left.\frac{\mathrm{d} \sigma_{c \bar{c}}^{\mathrm{NN}}}{\mathrm{d} y}\right|_{y=0}=175 \pm 13$ (stat.) \pm 23 (sys.) $\mu \mathrm{b}$. We assumed the same $f_{\text {frag }}(0.565 \pm 0.032)$ as in $\mathrm{p}+\mathrm{p}$ collisions. $d Y$ is obtained from (1) and $\sigma^{\text {inel }}=42 \mathrm{mb}$ is the total inelastic cross section [20]. The $N_{\text {bin }}$ is the number of binary collisions. $\mathrm{d} \sigma_{c \bar{c}}^{\mathrm{NN}} /\left.\mathrm{d} y\right|_{y=0}$ as a function of $N_{\text {bin }}$ is shown in the right panel of Fig. 6. Within errors, the results are in agreement and follow the number-of-binary-collisions scaling, which indicates that charm quark is produced via initial hard scatterings at early stage of the collisions at RHIC.

Differences in the kinematic distributions of particles between high-energy heavy-ion collisions and $\mathrm{p}+\mathrm{p}$ collisions can be quantified by the nuclear modification factor $R_{\mathrm{AA}}$ whose deviation from unity carries information about the properties of the QGP and its interaction with particles under study. The $R_{\mathrm{AA}}$ was calculated as

$$
R_{\mathrm{AA}}\left(p_{T}, N_{\mathrm{bin}}\right)=\frac{d Y\left(p_{T}, N_{\text {bin }}\right)}{N_{\text {bin }} f_{\text {Levy }}\left(p_{T}\right)}
$$

where the $f_{\text {Levy }}\left(p_{T}\right)$ represents the $\mathrm{p}+\mathrm{p}$ reference. $d Y\left(p_{T}, N_{\text {bin }}\right)$ together with $N_{\text {bin }} f_{\text {Levy }}\left(p_{T}\right)$ are shown in the left panel of Fig. 5 and $R_{\mathrm{AA}}\left(p_{T}, y\right)$ in panels a), b), and c) of Fig. 5 corresponding to different $N_{\text {bin }}$ revealing strong suppression in the most central collisions for $p_{T}>2 \mathrm{GeV} / \mathrm{c}$. Our $R_{\mathrm{AA}}\left(p_{T}\right)$ are compared to various model calculations1 [21-25]. We find that model calculations including a substantial amount of charm-medium interaction and hadronization through both fragmentation and coalescence can describe the data. The charm-medium interaction is needed for the suppression at large $p_{T}$, while coalescence could be important for the enhancement at $p_{T}$ around $1.3 \mathrm{GeV} / c$.

More recently, STAR has measured $R_{\mathrm{AA}}$ of $D^{0}$ meson in U+U collisions at ${\sqrt{s_{\mathrm{NN}}}}=193 \mathrm{GeV}$, where the Bjorken energy density is predicted to be about $20 \%$ higher than that in the same centrality interval in $\mathrm{Au}+\mathrm{Au}$ collisions [26]. The suppression of $D^{0}$ meson production at large $p_{T}$ in $\mathrm{Au}+\mathrm{Au}$ and $\mathrm{U}+\mathrm{U}$ collisions follows a global trend as a function of the number of participant nucleons in the collisions, $\left\langle N_{\text {part }}\right\rangle$ and is consistent with that measured for $\pi^{ \pm}$, as can be seen in the left panel of Fig. 6.

\section{Conclusions and Outlook}

STAR has measured open heavy-flavor production in $p+p, A u+A u$ and $U+U$ collisions. The measured production cross sections of charm quarks in $\mathrm{p}+\mathrm{p}$ collisions are consistent with perturbative QCD calculations. Model calculations including a substantial amount of charm-medium interaction 

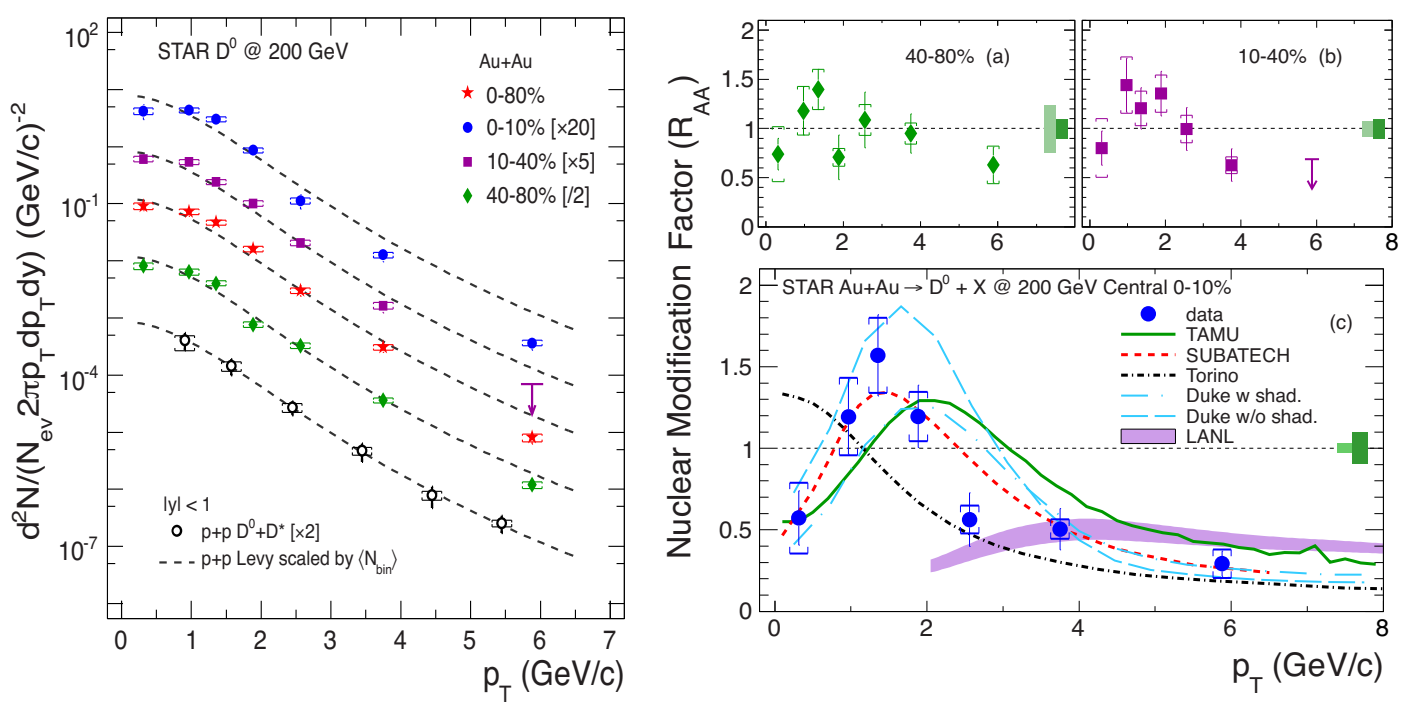

Figure 5. Left Panel: Centrality dependence of the $D^{0} p_{T}$ differential invariant yield in Au+Au collisions (solid symbols). The curves are number-of-binary-collision-scaled Levy functions from fitting to the $p+p$ result (open circles) [14]. The arrow denotes the upper limit with $90 \%$ confidence level of the last data point for 10-40\% collisions. The systematic uncertainties are shown as square brackets. Right panel: $D^{0}$ nuclear modification factor $R_{\mathrm{AA}}$ as a function of $p_{T}$ for most peripheral (panel a), semi-central (panel b), and the most central (panel c) $\mathrm{Au}+\mathrm{Au}$ collisions with theoretical predictions [21-25]. Green rectangles around unity represent systematic uncertainties of $N_{\text {bin }}$ uncertainty and $\mathrm{p}+\mathrm{p}$ normalization error.
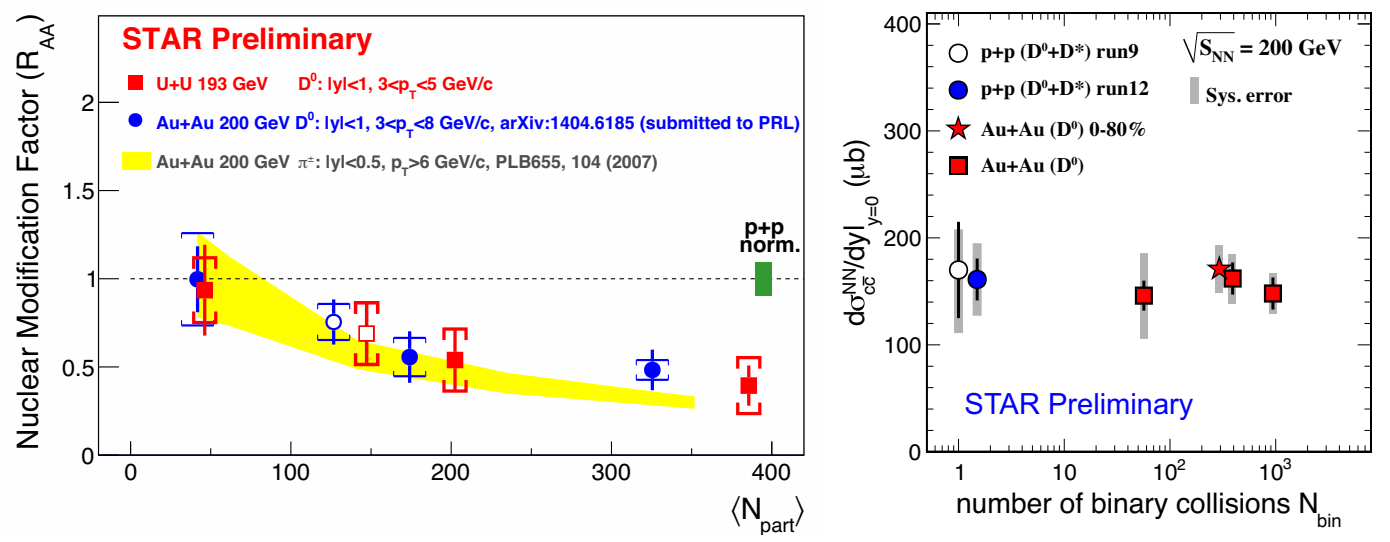

Figure 6. Left Panel: Nuclear modification factor $R_{\mathrm{AA}}$ as a function of the number of participant nucleons $\left\langle N_{\text {part }}\right\rangle$ in $\mathrm{Au}+\mathrm{Au}$ or $\mathrm{U}+\mathrm{U}$ collisions for $D^{0}$ with $p_{T}>3 \mathrm{GeV} / c$. Right panel: The charm production cross section per $N_{\text {bin }}$ as a function of $N_{\text {bin }}$. 
and hadronization via both fragmentation and coalescence describe the measured $D^{0}$ meson nuclear modification factor $R_{\mathrm{AA}}$. STAR has been recently upgraded with two new detectors, the Heavy Flavor Tracker (HFT) [27] and Muon Telescope Detector (MTD) [28]. The HFT is based on cuttingedge silicon detector technologies with excellent position resolutions and low material budgets. It provides STAR with the capability of identifying heavy-flavor particles and distinguishing between charm and bottom on an event-by-event basis through track impact parameter measurements [29]. The MTD enables STAR to identify high- $p_{T}$ muons for the first time, which is important for quarkonium measurements in di-muon decay channels and for open heavy-flavor measurements through e. g. electron-muon correlations. With the additions of the HFT and MTD, STAR is in an excellent position for heavy-flavor measurements with unprecedented precision in the coming years.

\section{Acknowledgements}

This work has been supported by the grant 13-02841S of the Czech Science Foundation (GACR) and by the Grant Agency of the Czech Technical University in Prague,

grant No. SGS13/215/OHK4/3T/14.

\section{References}

[1] B. Muller, Nucl. Phys. A 750, 84 (2005)

[2] Yu. L. Dokshitzer and D.E. Kharzeev, Phys. Lett. B 519, 199 (2001)

[3] B. I. Abelev et al., Phys. Rev. Lett. 98, 192301 (2007)

[4] S. S. Adler et al., Phys. Rev. Lett. 96, 032301 (2006)

[5] B. I. Abelev et al., J. High Energy Phys. 09, 112 (2012)

[6] M. Djordjevic, M. Gyulassy and S. Wicks, Phys. Rev. Lett. 94, 112301 (2005)

[7] A. Adil and I. Vitev, Phys. Lett. B 649, 139 (2007)

[8] He et al., Phys. Rev. C 86, 014903 (2012)

M. He et al., Phys. Rev. Lett. 110, 112301 (2013)

[9] P. B. Gossiaux et al., J. Phys. G 37, 094019 (2010)

P. B. Gossiaux et al., Nucl. Phys. A 904-905, 992c (2013)

[10] B. I. Abelev et al., Phys. Rev. C 75, 064901 (2007)

[11] B. I. Abelev et al., Phys. Rev. Lett. 97, 152301 (2006)

[12] J. Beringer et al., Phys. Rev. D 86, 010001 (2012)

[13] L. Adamczyk et al., Phys. Rev. Lett. 113, 72301 (2014)

[14] L. Adamczyk et al., Phys. Rev. D 86, 072013 (2012)

[15] M. Shao et al., Nucl. Instrum. Methods A 499, 624 (2003).

[16] J. Adams et al., Phys. Rev. Lett. 94, 062301 (2005).

[17] J. Adams et al. [STAR Collaboration], Phys. Rev. Lett. 91, 172302 (2003)

[18] M.Cacciari, P.Nason, and R.Vogt, Phys. Rev. Lett. 95, 122001 (2005)

[19] R. Vogt, private communication, (2012).

[20] M. Honda et al., Phys. Rev. Lett. 70, 525 (1993)

[21] M. He, R. J. Fries, R. Rapp, arXiv: 1204.4442.

[22] P. B. Gossiaux, J. Aichelin, M. Bluhm, T. Gousset, M. Nahrgang, S. Vogel, K. Werner, arXiv: 1207.5445 . 
[23] W. M. Albericoetal., Euro. Phys. J 71, 1666 (2011)

W. M. Albericoetal., Euro. Phys. J.73, 2481 (2013)

[24] S. S. Cao, G. Y. Qin and S. A. Bass, Phys. Rev. C 88, 044907 (2013)

[25] R. Sharma, I. Vitev and B. W. Zhang, Phys. Rev. C 80, 054902 (2009)

[26] D. Kikola, G. Odyniec and R. Vogt, Phys. Rev. C 84, 054907 (2011)

[27] Technical Design Report: The STAR Heavy Flavor Tracker (2011)

[28] L. Ruan et al., J. Phys. G: Nucl. Part. Phys. 36, 095001 (2009)

[29] Y.Zhang et al., J. Phys. G: Nucl. Part. Phys. 41, 025103 (2014) 